\title{
Modelo de gestão em bancos de olhos e seu impacto no resultado destas organizações
}

\author{
Management performance of eye banks and its \\ impact on those organizations results
}

Christiana Velloso Rebello Hilgert', Élcio Hideo Sato²

\section{ResUmo}

Objetivo: Avaliar a eficácia do modelo de gestão adotado pelo Banco de Olhos de Sorocaba (BOS) e Banco de Olhos da Santa Casa de Campo Grande (BOSC), segundo os critérios do Prêmio Nacional de Gestão em Saúde (PNGS) e demonstrar as características comuns e discordantes entre os dois Bancos de Olhos e um paralelo entre a efetividade do modelo de gestão adotado e o resultado destas organizações na doação de córneas. Métodos: Foi aplicado o questionário “Como estamos progredindo?", disponibilizado pela Fundação Malcolm Baldrige, composto por 40 questões fechadas agrupadas nos sete critérios do PNGS (Liderança, Planejamento Estratégico, Foco no Cliente e no Mercado, Foco em Recursos Humanos, Critério Gestão de Informações, Gestão de Processos, e Resultados) em toda a força de trabalho das duas instituições (21 funcionários do BOS e 8 do BOSC). Os resultados foram analisados utilizando o Teste Exato de Fischer e Teste não-paramétrico U de Mann-Whitney para comparação das médias entre as duas instituições. Resultados: Foram entrevistados 21 funcionários do BOS e 8 funcionários do $\mathrm{BOSC}$ ). O teste de Mann-Whitney mostrou uma maior concordância às proposições do questionário pelos respondentes do BOS em 6 dos 7 itens estudados (Liderança, Planejamento Estratégico, Foco no Cliente e no Mercado, Foco em Recursos Humanos, Gestão de Processos, e Resultados). Não houve diferença estatisticamente significativa no Critério Gestão de Informações. A maior diferença entre as duas instituições foi encontrada no critério Gestão de Processos $(\mathrm{p}<0.001)$. Conclusão: O modelo de gestão presente no BOS apresenta aspectos distintos e estatisticamente significantes comparados ao BOSC, em 6 dos 7 critérios estudados pelo questionário aplicado. O BOS é o que mais se aproxima de conceitos internacionalmente aceitos de excelência em gestão baseados nos critérios do PNGS do que o BOSC, sendo um dos motivos prováveis do resultado positivo no número de doações de córnea.

Descritores: Bancos de olhos/organização \& administração; Transplante de córnea, Gestão em saúde; Avaliação em saúde

\footnotetext{
${ }^{1}$ Instituto Hilton Rocha - Belo Horizonte (MG), Brasil;

${ }^{2}$ Instituto da Visão da Universidade Federal de São Paulo - (UNIFESP) São Paulo (SP), Brasil.
}

Os autores declaram inexistir conflitos de interesses

Recebido para publicação em 22/12/2010 - Aceito para publicação em 19/10/2011 


\begin{abstract}
Objective: To analyse the management of Sorocaba Eye Bank (BOS) and Santa Casa de Campo Grande Eye Bank (BOSC) with distinct results regarding number of donations using Prêmio Nacional Gestão em Saúde (PNGS) criteria for performance excellence.Methods: All the personel of the two eye banks were submitted to an interview using the 'Are We Making Progress?' questionnaire from Malcolm Baldrige Quality Program. The questionnaire items were organized by the seven criteria categories, as the PNGS's (Leadership, Strategic Planning, Customer and Market Focus, Measurement, Analysis and Knowledge Management, Human Resource Focus, Process Management, Bussiness Results). The findings were studied upon Fischers' exact test and Non parametric U test (Mann-Whitney) to compare the medium scores between the two Institutions. Results: Twenty-one employees from BOS and 08 from BOSC were submitted to the questionnaire. The Mann-Whitney test showed a higher level of agreement to the questionnaire proposals from BOS than the ones from BOSC in 6 out from the 7 items studied (Leadership, Strategic Planning, Costumer and Market Focus, Human Resource Focus, Process Management, Bussiness Results). There wasn't any statisticaly significant difference related to the criteria Measurement, Analysis and Knowledge Management. The highest difference on the concordance level between the two organizations was related to Process Management $(\mathrm{p}<0.001)$. Conclusion: The BOS management performance has distinct and statistically significant aspects compared to BOSC, shown on 6 out of 7 criteria studied by the questionnaire used. These data showed that the BOS performance management is closer to internationally accepted concepts of performance excellence, based on both PNGS Criteria and Malcolm Baldrige Quality Program, than BOSC.
\end{abstract} evaluation

Keywords: Eye banks/organization \& administration; Corneal transplant; Health management; Health

\section{INTRODUÇÃO}

$\mathbf{0}$ Censo Demográfico do Instituto Brasileiro de Geografia e Estatística (IBGE) de 2000 apurou que a deficiência visual é a deficiência física mais citada no Brasil, com 16,5 milhões de brasileiros afetados numa população de 170 milhões de habitantes $^{(1)}$. As doenças corneanas são a segunda causa de cegueira reversível no mundo, com cerca de 10 milhões de pessoas acometidas ${ }^{(2,3)}$. Para ser transplantada, a córnea deve ser retirada do doador preferencialmente até 6 horas depois de seu falecimento(4). No Brasil, a principal indicação de transplante de córnea é o ceratocone, com porcentagem que varia de $20 \%$ a $65 \%{ }^{(5,6)}$. Os Bancos de Olhos (BO) tem responsabilidade de captar, processar, avaliar, classificar, armazenar e distribuir tecidos oculares e devem atender às exigências legais para sua instalação e autorização de funcionamento.

Para avaliar seu sistema de gestão e alavancar a excelência em seus produtos e serviços, empresas brasileiras estão, cada vez mais, aplicando critérios que visam à melhoria da qualidade na organização como um todo. Neste sentido, o Prêmio Nacional da Qualidade (PNQ), instituído em 1992, busca promover o entendimento dos requisitos para alcançar a excelência do desempenho e troca de informações sobre métodos e sistemas de gestão( ${ }^{(7,8)}$.
Administrado pela Fundação para o Prêmio Nacional da Qualidade (FPNQ), o PNQ surgiu do esforço de um grupo de profissionais das áreas industriais, consultoria e acadêmica que se dedicou ao trabalho de pesquisa e análises de diversas premiações, em consonância com o que ocorre em outros países, em particular nos Estados Unidos (Prêmio Malcolm Baldrige) e no Japão (Prêmio Deming), com o intuito de formular fundamentos, critérios e o processo de premiação. Todo ano a FPNQ revisa, atualiza e divulga os critérios de excelência do $\mathrm{PNQ}^{(7-9)}$.

A versão PNQ vigente na época do estudo constava de sete critérios: liderança; planejamento estratégico; foco no cliente e no mercado; informação e análise; gestão de pessoas; gestão de processos; e resultados da organização. O PNQ se apóia em fundamentos que permeiam os sete critérios: enfoques e desdobramentos sobre a qualidade centrada no cliente; comprometimento da alta direção; valorização das pessoas; responsabilidade social; visão de futuro de longo alcance; foco nos resultados; aprendizado contínuo; gestão baseada em fatos e em processos; pró-atividade e resposta rápida ${ }^{(7,8)}$.

O Prêmio Nacional da Gestão em Saúde (PNGS), baseado em critérios do PNQ, foi criado por iniciativa do Controle de Qualidade Hospitalar $(\mathrm{CQH})$ para estimular organizações da área da saúde a avaliar e buscar melhorias contínuas de seus sistemas de 
gestão, reconhecendo aquelas organizações que se destacam pela utilização de práticas de gestão e que apresentem resultados superiores de desempenho. Sua visão é servir como modelo referencial para avaliação e orientação da gestão das organizações de saúde do Brasil, assim como a Fundação Malcolm Baldrige nos Estados Unidos, que desde 1987 reconhece e premia empresas detentoras de cultura organizacional voltada para a excelência ${ }^{(9-12)}$.

\section{Métodos}

Estudo foi realizado com a autorização do Hospital de Olhos de Sorocaba, no BOS e da Santa Casa de Campo Grande, no BOSC, seguindo normas administrativas das duas instituições, segue os princípios básicos contidos na Declaração de Helsinque de 1964 e foi submetido à análise e aprovação do Comitê de Ética em Pesquisa da Universidade Federal de São Paulo UNIFESP (CEP N 1019/04)

O questionário "Como estamos progredindo?" (Anexo 1) foi traduzido e adaptado para o portuguêsbrasileiro do questionário "Are wemakingprogress? 2003". Este questionário é disponibilizado pela Fundação Malcolm Baldrige com a finalidade de avaliar a efetividade de modelos de gestão segundo os sete critérios de avaliação dos prêmios Malcolm Baldrige nos Estados Unidos e PNQ/ PNGS no Brasil ${ }^{(9,13)}$.(FNPQ, 2002b; NIST, 2006). Foi aplicado por entrevistador treinado para tal em toda a equipe de trabalho envolvida no processo de doação de córneas dos dois BO, no ano de 2004.

O questionário é composto por 40 questões fechadas, que estão agrupadas segundo os sete critérios do PNGS:

- Liderança - 7 questões;

- Planejamento Estratégico - 3 questões;

- Foco no cliente e no mercado - 5 questões;

- Medidas, análises e gestão de informações - 6 questões;

- Foco nos recursos humanos - 6 questões;

- Gestão de Processos - 4 questões;

- Resultados da Organização - 9 questões .

Há um número variável de proposições acerca do modelo de gestão empregado, para cada um dos sete critérios com as quais o respondente pode: concordar plenamente, concordar, ser indiferente, discordar ou discordar totalmente. Quanto mais concordante for a resposta, mais próxima da excelência o modelo se encontra. A equipe de pesquisa ( 2 pessoas externas) recebeu treinamento para entender o propósito do estudo. A confidencialidade do entrevistado foi garantida, através da não identificação do entrevistado e todas as entrevistas foram realizadas individualmente para que não houvesse qualquer viés de resposta ou constrangimento do entrevistado ao responder alguma questão.

Para avaliar a importância do modelo de gestão adotado em um BO como fator no resultado, foram selecionados o BOS, pela sua importância no cenário brasileiro de doações e transplante de córnea e o BOSC, em Mato Grosso do Sul, o qual, instalado em um hospital geral, apresenta ainda um pequeno, mas crescente número de doações frente a seu potencial e que representaria a realidade da maioria dos $\mathrm{BO}$ em funcionamento no Brasil.

O BOS foi fundado em 1971, contando com a colaboração efetiva de Clubes de Serviços, Lojas Maçônicas, Sindicatos e Associações de Classe. Em 1984 foi implantada uma administração profissionalizada na entidade no tocante à arrecadação e distribuição das córneas $^{(14,15)}$.

O BOSC, até a época da regulamentação oficial feita pelo Ministério da Saúde em 1999, funcionava informalmente, contando com ações voluntárias e o esforço pessoal das partes interessadas: médicos, pacientes e pessoal de apoio. A doação de córneas para transplante é feita em Campo Grande, Mato Grosso do Sul, desde 1987. É o único BO ativo em todo o estado do Mato Grosso do Sul. Pertence à Sociedade Beneficente Campo Grande - Santa Casa, um hospital filantrópico sem fins lucrativos de grande porte (840 leitos) e funciona como um setor do hospital, subordinado à Comissão Intra-Hospitalar de Doação de Órgãos e Tecidos para Transplante (CIHDOTT)da entidade. Não possui missão ou visão definida.

Inicialmente, todas as variáveis sóciodemográficas como sexo, idade, tempo de serviço no BO, escolaridade e as respostas dos itens dos questionários foram analisados descritivamente através das frequências absolutas e relativas. Em seguida, foram realizados testes exatos de Fisher para avaliar se os perfis sócio-demográficos dos respondentes e a distribuição das opiniões a respeito dos diversos quesitos colocados eram semelhantes nas duas instituições.

Para os escores de cada um dos sete critérios, a análise foi realizada através das seguintes medidas/resumo: mínimo, máximo, média, quartis e desvio padrão. Para verificar se a opinião dos funcionários a respeito dos sete critérios foi semelhante ou não entre as duas entidades, realizou-se o teste não paramétrico U de MannWhitney para comparação de médias. Nos testes estatísticos foi utilizado um nível de significância de 5\%. 


\section{Resultados}

Foram entrevistados 29 funcionários das duas instituições, dos quais 21 (11 homens, 10 mulheres, média de idade $30,57 \pm 10,01$ anos) trabalhavam no BOS e 8 ( 2 homens, 6 mulheres, média de idade 31,50 7,76 anos), no BOSC. Dos respondentes do BOS, 52,4\% eram homens, já no BOSC 75\% eram mulheres. Nos BO das duas instituições há predominância de funcionários jovens, especificamente entre 20 e 30 anos - BOS $(57,1 \%)$, $\operatorname{BOSC}(62,5 \%)$. Quanto à escolaridade no BOS, $19 \%$ e no BOSC, $50 \%$ tinham curso superior incompleto. O BOS apresenta funcionários com maior tempo de serviço, sendo que o grupo mais expressivo de funcionários estava de 1 a 3 anos na empresa (61,9\%). Já o BOSC apresenta o maior quadro de funcionários no grupo com menos de 1 ano na Instituição $(62,5 \%)$. Em relação ao conhecimento da missão da empresa, no BOS, $100 \%$ e no BOSC, $75 \%$ dos respondentes a conheciam. No critério liderança, enquanto no BOS a maioria dos funcionários acredita que suas lideranças utilizam os valores da empresa em suas gestões ( $81 \%$ ), no BOSC nota-se respostas bem distribuídas, sendo que $37,5 \%$ dos funcionários são indiferentes, $50 \%$ acreditam e $12,5 \%$ não acreditam que suas lideranças utilizam os valores da empresa em suas gestões. No BOS, $90,5 \%$ dos funcionários concordam que a liderança cria um ambiente de trabalho que os ajudam a desempenhar suas funções. Já no BOSC, 50\% acreditam, porém $37,5 \%$ são indiferentes e $12,5 \%$ não acreditam que seus líderes criam um ambiente de trabalho que os ajudam a executar suas funções.

No critério Foco no Cliente e no Mercado, a maioria dos funcionários das duas Instituições tem conhecimento dos clientes importantes (potenciais doadores e familiares): No BOS: $76,2 \%$ e no BOSC: $87,5 \%$. No BOS grande parte dos funcionários mantém contato com seus clientes $(66,7 \%)$, no BOSC a maior parte dos funcionários não demonstra estabelecer contato (66,2\%). Dos funcionários do BOS, $76,2 \%$ referem que seus clientes dizem o que necessitam e desejam, enquanto que no BOSC esta porcentagem é de $37,5 \%$. No BOS, a maior parte dos funcionários $(66,7 \%)$ questiona seus clientes para saber se estão satisfeitos ou não com o trabalho oferecido e no BOSC, apenas 12,5\% afirmam questioná-los. Dos funcionários do BOS, 66,6\% afirmam ter permissão para tomar decisões que solucionem problemas para os seus clientes. No BOSC 37,5\% afirmam ter permissão para tal.

A Tabela 1 mostra um resumo das medidas de média, erro padrão, desvio padrão, variância, mínimo, máximo, $1^{\circ}$ quartil, mediana e $3^{\circ}$ quartil para as duas
Instituições avaliadas. $\mathrm{O}$ teste de Mann-Whitney mostrou haver diferença estatística em 6 dos 7 critérios estudados entre as duas Instituições. Apenas no critério Medidas, Análise e Gestão de Informações $(p=0,102)$ não houve diferenças estatisticamente significativas entre elas. A mediana de escore para os sete critérios (Liderança, Planejamento Estratégico, Foco no Cliente e no Mercado, Medidas, Análise e Gestão de Informações, Foco nos Recursos Humanos, Gestão de Processos e Resultados da Organização) foi maior no BOS que no BOSC

\section{DısCUSSÃO}

Nas duas instituições estudadas, a análise dos perfis sócio-demográfico dos respondentes se mostrou semelhante. Deste modo, considerando que os aspectos sócio-demográficos poderiam ter pouca influência na discrepância de resultados entre os BO estudados, a decisão de aplicar benchmarking (busca e a implantação das melhores práticas) ${ }^{(16)}$, comparando os $\mathrm{BO}$ que apresentem organização e resultados distintos, é muito apropriada.

A avaliação do Modelo de Gestão adotado por estas duas Instituições se baseou nos fundamentos e critérios do PNQ e do PNGS e os resultados buscaram demonstrar se a distribuição das opiniões a respeito das diversas questões colocadas era semelhante.

No critério Liderança, o BOS teve respostas mais concordantes do que o BOSC. Dos itens que fazem parte da avaliação desse critério, observam-se distribuições de opiniões diferentes entre os respondentes das duas instituições que poderiam refletir uma lacuna quanto à capacidade de estimular a motivação nas pessoas e a habilidade de comunicação, o que seria a força propulsora da excelência organizacional, em que a alta direção mantém o engajamento das pessoas em todos os níveis, na causa da organização $^{(7)}$. O item Q1a ("Conheço a missão da minha empresa") se destaca positivamente no BOS, visto que $100 \%$ dos respondentes relataram ter conhecimento dos valores da instituição contra $75 \%$ no BOSC.

Dos cinco itens que fazem parte da avaliação do critério Foco no Cliente e no Mercado (Gráficos 1 e 2 ), observa-se opiniões diferentes entre os respondentes das duas instituições nos itens Q3d ("Eu pergunto se meus clientes estão satisfeitos ou não com meu trabalho") e Q3e ("Eu tenho permissão para tomar decisões que solucionem problemas para os meus clientes."); no BOS percebe-se uma maior concordância nesse critério e no BOSC é observada uma menor autonomia frente a decisões em relação aos clientes. A qualidade é intrínseca ao produto, porém o cliente, no caso o responsável pela do- 
ação, é quem julga a partir de suas próprias percepções. Estas percepções se formam por meio de características e atributos, que adicionam valor para os clientes, intensificam sua satisfação, determinam suas preferências e os tornam fiéis à organização ${ }^{(12)}$.

Um estudo francês mostra que, após a alteração da regulamentação da doação de córneas naquele país em que se tornou necessária a autorização da família do doador para a remoção de córneas, houve um decréscimo significativo nos índices de transplantes realizados ${ }^{(17)}$. Outro aspecto citado é que os maiores índices de resposta positiva à doação de córneas foram obtidos quando a abordagem foi realizada por um grupo multidisciplinar treinado para $\operatorname{tal}^{(18)}$. Portanto, o conhecimento das necessidades atuais e futuras dos clientes, o doador ou seus familiares, é o ponto de partida na busca da excelência do desempenho da organização.

Dos seis itens que fazem parte da avaliação do critério Foco nos Recursos Humanos, observa-se distribuição de opiniões diferentes entre os respondentes das duas instituições nos dois itens: Q5a ("Eu posso realizar mudanças que melhorarão o meu trabalho.") e Q5c ("Meu chefe me encoraja a desenvolver minhas habilidades profissionais de forma que eu possa avançar em minha carreira."). Nesta distribuição, a maior concordância ocorre, novamente, no BOS e a concordância tendendo a indiferença no BOSC.

A valorização das pessoas leva em consideração a diversidade de anseios e necessidades que, uma vez identificados e utilizados na definição das estratégias, dos planos e das práticas de gestão organizacionais, promovem o desenvolvimento, o bem estar e a satisfação da força de trabalho, a atração e retenção de talentos humanos, bem como um clima organizacional participativo e agradável, possibilitando o alcance do alto desempenho da organização e o crescimento das pessoas. A profissionalização das equipes de abordagem requer intenso treinamento, entretanto, o resultado é logo percebido quando estudos mostraram o aumento nas doações de córnea obtido devido ao seu trabalho ${ }^{(14,19)}$.

Numa segunda análise os resultados não são rela-

Tabela 1

Medidas dos escores e teste de Mann-Whitney

\begin{tabular}{|c|c|c|c|c|c|c|c|c|}
\hline Instituição & $\begin{array}{l}\text { Medidas- } \\
\text { Resumo }\end{array}$ & Liderança & $\begin{array}{c}\text { Planej. } \\
\text { Estratégico }\end{array}$ & $\begin{array}{c}\text { Foco no } \\
\text { cliente } \\
\text { e no } \\
\text { mercado }\end{array}$ & $\begin{array}{c}\text { Medidas, } \\
\text { análises e } \\
\text { gestão de } \\
\text { informações }\end{array}$ & $\begin{array}{c}\text { Foco nos } \\
\text { recursos } \\
\text { humanos }\end{array}$ & $\begin{array}{l}\text { Gestão de } \\
\text { Processos }\end{array}$ & $\begin{array}{l}\text { Resultados } \\
\text { da } \\
\text { organização }\end{array}$ \\
\hline
\end{tabular}

Sorocaba

\begin{tabular}{|c|c|c|c|c|c|c|c|}
\hline Média & 4,17 & 3,71 & 3,78 & 4,03 & 4,07 & 4,13 & 4,13 \\
\hline Erro Padrão & 0,12 & 0,18 & 0,19 & 0,12 & 0,12 & 0,12 & 0,08 \\
\hline Desvio Padrão & 0,57 & 0,82 & 0,85 & 0,54 & 0,54 & 0,56 & 0,39 \\
\hline Variância & 0,33 & 0,67 & 0,72 & 0,29 & 0,30 & 0,31 & 0,15 \\
\hline Mínimo & 3,14 & 2,00 & 2,20 & 3,00 & 3,33 & 2,75 & 3,33 \\
\hline Máximo & 5,00 & 5,00 & 5,00 & 5,00 & 5,00 & 5,00 & 5,00 \\
\hline $1^{\circ}$ Quartil & 3,64 & 3,33 & 3,20 & 3,67 & 3,50 & 4,00 & 3,89 \\
\hline Mediana & 4,14 & 3,67 & 4,00 & 4,00 & 4,00 & 4,00 & 4,00 \\
\hline $3^{\circ}$ Quartil & 4,57 & 4,17 & 4,30 & 4,42 & 4,42 & 4,38 & 4,50 \\
\hline $\mathrm{N}$ & 21 & & & & & & \\
\hline
\end{tabular}

Santa Casa de Campo Grande

$\begin{array}{lccccccc}\text { Média } & 3,54 & 2,79 & 2,85 & 3,67 & 3,40 & 3,31 & 3,69 \\ \text { Erro Padrão } & 0,15 & 0,36 & 0,24 & 0,13 & 0,28 & 0,20 & 0,12 \\ \text { Desvio Padrão } & 0,42 & 1,01 & 0,69 & 0,38 & 0,79 & 0,58 & 0,33 \\ \text { Variância } & 0,18 & 1,01 & 0,48 & 0,14 & 0,62 & 0,33 & 0,11 \\ \text { Mínimo } & 2,86 & 1,00 & 1,60 & 3,17 & 2,17 & 2,25 & 3,33 \\ \text { Máximo } & 4,14 & 4,00 & 3,60 & 4,33 & 4,33 & 3,75 & 4,22 \\ 1^{\circ} \text { Quartil } & 3,21 & 1,92 & 2,40 & 3,33 & 2,67 & 2,81 & 3,44 \\ \text { Mediana } & 3,50 & 3,00 & 3,00 & 3,75 & 3,42 & 3,63 & 3,56 \\ 3^{\circ} \text { Quartil } & 3,93 & 3,58 & 3,50 & 3,83 & 4,13 & 3,75 & 4,00 \\ \text { N } & 8 & & & - & & & \\ \text {-Whitney } & 32,00 & 40,50 & 34,00 & 50,50 & 40,00 & 13,50 & 36,00 \\ & 0,009 & 0,031 & 0,012 & 0,102 & 0,030 & <0,001 & 0,017\end{array}$




\section{Gráfico 1}

Distribuição das respostas para as cinco questões contidas no critério Foco no Cliente e no Mercado para o BOS

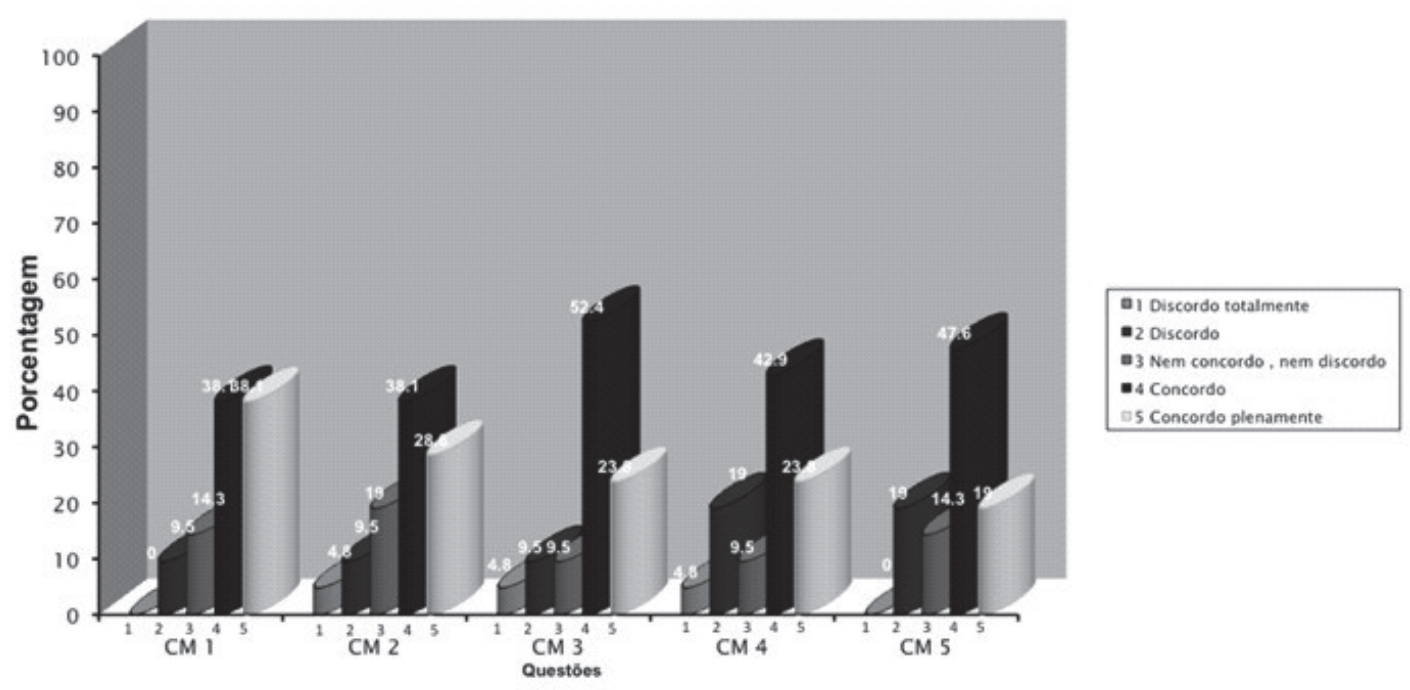

Gráfico 2

\section{Distribuição das respostas para as cinco questões contidas} no critério Foco no Cliente e no Mercado para o BOSC
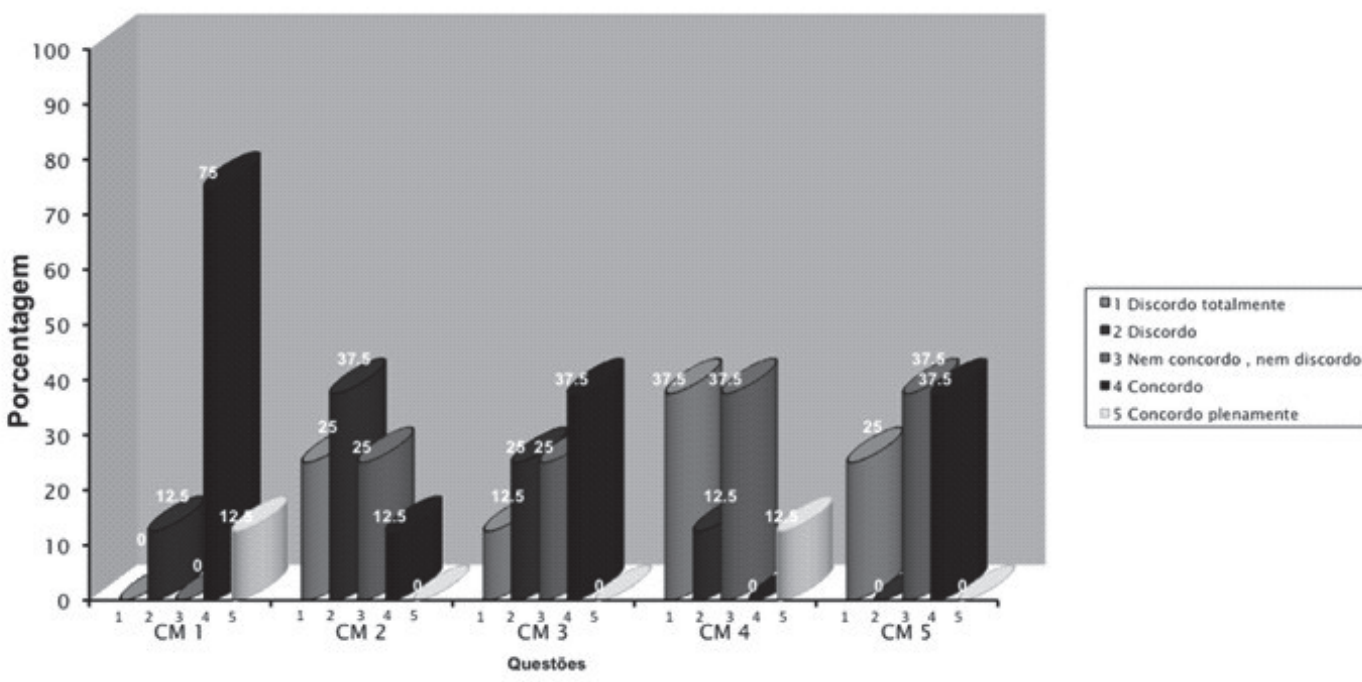

cionados com a distribuição das respostas nas 40 questões, e sim quanto à concordância dos $\mathrm{BO}$ em relação aos sete critérios, comparando-os e mostrando sua tendência à excelência em gestão. O estudo estatístico aplicado (Tabela 1) demonstra uma diferença estatisticamente significativa, em que os funcionários do BOS apresentam um nível de concordância com as proposições do questionário maior que os do $\mathrm{BOSC}$ em seis dos sete critérios estudados (liderança, planejamento estratégico,foco no cliente e no mercado, foco nos recursos humanos, gestão de processos e resultados da organização). A diferença alcançou níveis maiores no critério gestão de processos, provavelmente em decorrência do processo de implantação da ISO 9001 em que se encontra o BOS, gerando avaliação favorável neste critério.

No BOSC podem-se destacar algumas caracterís- 


\section{Anexo 1 - Questionário "Como estamos progredindo?"}

\section{Critério 1 - Liderança}

1a- Conheço a missão da minha empresa?

1b- Nossos líderes e chefes utilizam os valores da nossa empresa para nos guiar?

1c- Nossos líderes criam um ambiente de trabalho que me ajuda a executar o meu trabalho?

1d- Os nossos líderes compartilham comigo informações sobre a nossa empresa?

1e- Os nossos líderes me encorajam a aprender o que pode me ajudar a crescer profissionalmente, em minha carreira?

1f- Minha empresa me informa o que julga ser mais importante?

1g- Minha empresa me pergunta o que penso?

\section{Critério 2 - Planejamento Estratégico}

2a- Minha empresa pesquisa minhas idéias quando faz planejamentos para o futuro?

2b- Eu sei quais projetos e planos da minha empresa que afetarão a mim e a meu trabalho?

2c- Eu sei dizer se estamos progredindo no que foi planejado para meu grupo de trabalho?

\section{Critério 3 - Foco no cliente e no mercado}

3a- Eu sei quais são os nossos clientes mais importantes-potenciais doadores e familiares?

3b- Eu mantenho contato constante com os meus clientes?

3c- Meus clientes me dizem o que eles precisam e querem?

3d- Eu pergunto se meus clientes estão satisfeitos ou não com meu trabalho?

3e- Eu tenho permissão para tomar decisões que solucionem problemas para os meus clientes?

\section{Critério 4 - Medidas, análises e gestão de informações.}

4a- Sei como medir a qualidade de meu trabalho?

4b- Sei como analisar a qualidade do meu trabalho já executado para avaliar se é necessário proceder a mudanças?

4c- Eu utilizo estas análises para tomar decisões relativas a meu trabalho?

4d- Sei como as medidas que uso em meu trabalho se adequam às medidas gerais existentes com vistas ao crescimento e aperfeiçoamento da empresa?

$4 \mathrm{e}-\mathrm{Eu}$ consigo todas as informações importantes que necessito para executar bem o meu trabalho?

4f- Eu consigo todas as informações que necessito saber sobre o desempenho da minha empresa?

\section{Critério 5 - Foco nos recursos humanos}

5a- Eu posso realizar mudanças que melhorarão o meu trabalho?

$5 \mathrm{~b}$ - As pessoas com quem trabalho cooperam e trabalham como um time?

5c- Meu chefe me encoraja a desenvolver minhas habilidades profissionais de forma que eu possa avançar em minha carreira?

$5 d-$ Sou reconhecido pelo meu trabalho?

$5 e-$ Tenho segurança e estabilidade no meu trabalho?

$5 f-$ Meu chefe e minha empresa se preocupam comigo?

\section{Critério 6 - Gestão de Processos}

6a- Consigo tudo que necessito para realizar o meu trabalho?

6b- Eu coleto dados e informações sobre a qualidade do trabalho que executo?

$6 \mathrm{c}-$ Nós temos bons processos para realizar nosso trabalho?

6d- Tenho controle sobre os meus processos de trabalho?

\section{Critério 7 - Resultados da Organização}

7a- Meus clientes estão satisfeitos com o meu trabalho?

$7 \mathrm{~b}$ - $\mathrm{O}$ produto do meu trabalho está dentro dos padrões requeridos?

7c- Eu sei se minha empresa está indo bem financeiramente?

7d- Minha empresa utiliza bem meu tempo e minhas habilidades?

7e- Minha empresa remove os obstáculos que possam prejudicar seu crescimento e progresso?

7f- Minha empresa obedece às leis e regulamentos?

$7 \mathrm{~g}$ - Minha empresa segue altos padrões de ética?

7h- Minha empresa me ajuda a realizar trabalhos comunitários?

7i- Estou satisfeito com o meu trabalho? 
ticas da instituição que se diferenciam do BOS como o tempo de existência ainda curto e a falta de identidade do $\mathrm{BO}$ em si como empresa, pois é subordinada à CIHDOTT de um hospital de grande porte cujo maior financiador é o SUS. Não sendo uma entidade autônoma, e dependente do hospital, os valores do BOSC se confundem com os valores da Santa Casa como um todo, e as decisões tomadas em relação ao BO são avaliadas dentro de um panorama mais amplo de saúde pública.

Além da implantação de um modelo de gestão adequado a cada realidade, inúmeros outros fatores contribuem para o aumento de doação de córneas com finalidade de transplante: a educação da sociedade e conscientização da população potencialmente doadora, campanhas de marketing, modelo de abordagem adequado, treinamento constante de equipes multidisciplinares ${ }^{(15,17,18,20,21)}$, assim como alterações na interface BO e SUS que visem dinamizar todo o processo e facilitar o seu custeio são medidas que podem ser implantadas para melhoria do BOSC.

\section{CONCLUSÃo}

O modelo de gestão presente no BOS apresenta aspectos distintos e estatisticamente significativos em relação ao BOSC. O modelo de gestão em funcionamento no BOS aproxima-se mais da excelência em gestão que o modelo em funcionamento no BOSC, segundo os critérios do PNGS, por ocasião deste estudo. A estrutura organizacional difere entre as duas instituições estudadas, evidenciada desde a sua fundação e histórico, seus valores, suas lideranças e relações institucionais, até o seu resultado final em número de doações de córnea.

\section{RefERÊNCIAS}

1. Instituto Brasileiro de Geografia e Estatística - IBGE. Censo Demográfico 2000. Tabulação avançada. Tabela 1.1.3. População residente, por situação de domicílio e sexo, segundo o tipo de deficiência - Brasil [ Internet]. Rio de Janeiro: IBGE; 2000 a. [citado 2006 Jul 10]. Disponível em: http://www.ibge.gov.br/home/estatistica/populacao/ censo2000/tabulacao_avancada/tabelabrasil1.1.3.shtm.

2. Whitcher, JP, Srinivasan M, Upadhyay MP. Corneal blindness: a global perspective. Bull World Health Organ. 2001;79(3):214-21.

3. World Health Organization. Media Centre. Magnitudes and causes of visual impairment [ Internet]. Geneva: WHO; 2002. [cited $2006 \mathrm{Jul}$ 10]. Available from:

htpp:// www.who.int/mediacentre/factsheets/fs282/en.

4. Sato EH. Transplante de córnea e bancos de olhos[ Internet]. Universo Visual. 2002;(2). [citado 2006 Jul 19]. Disponível em: ht tp://www.universovisual.com.br/publisher/ preview.php?edicao=0602\&id_mat $=120$.

5. Thomaz A, Ando E, Akaishi L, Barros AC. Rejeição em transplante de córnea. RevBras Oftalmol. 1990;49(6):15-20.
6. Barros CR, Oliveira DF, Castro RS, Lima VMP, Kara José N. Incidência e falência em transplante de córnea [resumo]. ArqBras Oftalmol. 1992;55(4):178.

7. Fundação para o Prêmio Nacional da Qualidade. Critérios de avaliação Prêmio Nacional de Gestão em Saúde. São Paulo: Fpnq; 2002.

8. Fundação Nacional da Qualidade - FNQ. 15 anos promovendo a excelência da gestão para o aumento da competitividade das empresas e do Brasil, 2005. [ Internet]. São Paulo, 2005. [citado 2006 Jul 1818]. Disponível em: http://www.fnq.org.br/ site/315/default.aspx.

9. National Institute of Standards and Technology - NIST. Baldrige National Quality Program. Are We Making Progress? [Internet]. [ cited 2006 Jul 18]. Available from: http:// www.quality.nist.gov/PDF_files/Progress.pdf.

10. Fundação para o Prêmio Nacional da Qualidade. Caso para estudo Hospital Taquara" [Internet]. São Paulo: Fpnq; 2000. [citado 2006 Jul 18]. Disponível em: http:// www.sebraepr.com.br/FCKeditor/userfiles/file/UAP/Prosperar/ OutrosMateriais/Taquaral\%202000\%20-\%20CE.pdf.

11. Scarpi MJ, organizador. Gestão de clínicas médicas. São Paulo: Futura: c2004.p. 21-156.

12. Prêmio Nacional da Gestão em Saúde -PNGS. Critérios de Avaliação, 2004.[Internet]. [citado 2006 Jul 20] Disponível e m: ht t p://www.cqh.org.br/pngs/files/pngs/ 2004_9_17_Criterios\%20PNGS\% 202004_Set_Rev_04.pdf.

13. Fundação para o Prêmio Nacional da Qualidade. Primeiros passos para a excelência: critérios para o bom desempenho e diagnóstico da organização. São Paulo: Fpnq; 2004. 59p.

14. Banco de Olhos de Sorocaba. Hospital Oftalmológico de Sorocaba. História [Internet]. [citado 2006 Set 5] Disponível em: http://www.bos.org.br/bos/index_bos.html.

15. Farias RJM, Sousa LB. Impacto do marketing dos processos de divulgação nas doações de córneas a um banco de tecidos oculares humanos e avaliação do perfil socioeconômico de seus doadores. ArqBras Oftalmol. 2008;71(1):28-33.

16. Camp Robert C. Benchmarking; o caminho da qualidade total; identificando, analisando e adaptando as melhores práticas da administração que levam a maximização da performance empresarial. $2^{\mathrm{a}}$ ed. São Paulo: Pioneira; 1995.

17. Muraine M, Menguy E, Eupherte L, Martin J, Pouille P, Sadorge T, Brasseur G. [An important step in cornea procurement: interview with the family of donors]. J FrOphtalmol. 1997;20(1):25-30. French.

18. Rodrigues AM, Sato E. Entendimento dos médicos intensivistas sobre o processo de doação de córneas. ArqBras Oftalmol. 2003;66(1):29-32.

19. The Foundation for the Malcolm Baldrige National Quality Award [Internet]. [cited 2006, Aug 17] Available from: www.baldrigefoundation.org/baldrige/index.html.

20. Kotler P. Administração de marketing. 5a ed. São Paulo: Atlas; 1998. p.45-7

21. Moreira KC. Avaliação do perfil dos pacientes e do tempo de espera no banco de olhos do hospital do Servidor Público de São Paulo [tese]. São Paulo: Universidade Federal de São Paulo. Escola Paulista de Medicina; 2003.

\author{
Endereço para correspondência: \\ Christiana Velloso Rebello Hilgert \\ Rua Rubens Gil de Camillo, 83 Chácara Cachoeira \\ CEP 79040090 - Campo Grande - (MS), Brasil \\ Tel:/fax: (67) 3026-1333 \\ Email: c.hilgert@terra.com.br
}

